\title{
BMJ Open Role of context in care transition interventions for medically complex older adults: a realist synthesis protocol
}

\author{
Kristen B Pitzul, Natasha E Lane, Teja Voruganti, Anum I Khan, Jennifer Innis, \\ Walter P Wodchis, G Ross Baker
}

To cite: Pitzul KB, Lane NE, Voruganti T, et al. Role of context in care transition interventions for medically complex older adults: a realist synthesis protocol. BMJ Open 2015;5:e008686. doi:10.1136/bmjopen-2015008686

- Prepublication history for this paper is available online. To view these files please visit the journal online (http://dx.doi.org/10.1136/ bmjopen-2015-008686).

Received 6 May 2015 Revised 21 August 2015 Accepted 21 September 2015

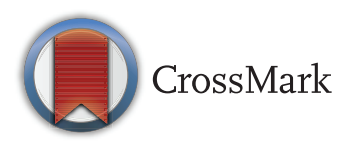

Institute of Health Policy, Management, and Evaluation, University of Toronto, Toronto, Canada

Correspondence to Kristen B Pitzul; Kristen. pitzul@mail.utoronto.ca

\section{ABSTRACT}

Introduction: Approximately $30-50 \%$ of older adults have two or more conditions and are referred to as multimorbid or complex patients. These patients often require visits to various healthcare providers in a number of settings and are therefore susceptible to fragmented healthcare delivery while transitioning to receive care. Care transition interventions have been implemented to improve continuity of care, however, current evidence suggests that some interventions or components of interventions are only effective within certain contexts. There is therefore a need to unpack the mechanisms of how and within which contexts care transition interventions and their components are effective. Realist review is a synthesis method that explains how complex programmes work within various contexts. The purpose of this study is to explain the effect of context on the activities and mechanisms of care transition interventions in medically complex older adults using a realist review approach.

Methods and analysis: This synthesis will be guided by Pawson and colleagues' 2004 and 2005 protocols for conducting realist reviews. The underlying theories of care transition interventions were determined based on an initial literature search using relevant databases. English language peer-reviewed studies published after 1993 will be included. Several relevant databases will be searched using medical subject headings and text terms. A screening form will be piloted and titles, abstracts and full text of potentially relevant articles will be screened in duplicate. Abstracted data will include study characteristics, intervention type, contextual factors, intervention activities and underlying mechanisms. Patterns in Context-ActivityMechanism-Outcome (CAMO) configurations will be reported.

Ethics and dissemination: Internal knowledge translation activities will occur throughout the review and existing partnerships will be leveraged to disseminate findings to frontline staff, hospital administrators and policymakers. Finalised results will be presented at local, national and international conferences, and disseminated via peer-reviewed publications in relevant journals.

\section{Strengths and limitations of this study}

- This review will highlight which components of care transition interventions work and how they operate within various contexts, rather than considering intervention effectiveness as a whole entity.

- Thorough retrieval of pertinent information about the intervention components, mechanisms and study context (ie, search for associated relevant publications and direct contact with study authors if required).

- Systematic screening protocol with all steps completed in duplicate.

- Study quality will not be formally assessed.

- Only English language studies will be included.

\section{INTRODUCTION}

By the year 2030, the percentage of persons over the age of 65 years is predicted to double, with an estimated $20-25 \%$ of the world's population being 65 years of age and older. ${ }^{1}$ Over $60 \%$ of adults aged 65 years and older have at least one chronic health condition, and approximately $30-50 \%$ have two or more conditions and are referred to as multimorbid or complex patients. $^{2}{ }^{3}$ These complex older adults use extensive healthcare resources and often require visits to various healthcare providers in a number of settings. ${ }^{2}$ 4-8 Evidence suggests that managing the transition between these providers and/or settings is difficult within current healthcare delivery systems, and that these transitions typically result in fragmented care. $^{4-79}$

Fragmented transitions can arise from a multitude of factors at the system (eg, lack of formal institutional relationships), provider (eg, incomplete discharge summaries) and patient (eg, transportation concerns) level. ${ }^{4}{ }^{10-15}$ It can also result in a multitude of adverse events, including medication errors, increased caregiver and patient burden, 
injuries, and increased hospital readmission rates. ${ }^{10}$ 16-22 Coleman and Boult ${ }^{4}$ defined transitional care as "set of actions designed to ensure the coordination and continuity of healthcare as patients transfer between different locations or different levels of care within the same location." In certain countries (ie, the UK) the care provided during transitions is also known as intermediate care. ${ }^{23}$ For the purpose of this study, interventions implemented to improve transitional care (ie, care transition interventions) are considered synonymous with the above definition of transitional care.

Previous evidence has primarily focused on the implementation of care transition interventions to improve continuity of care for patients discharged from hospital to either home, home with care or another institution. $^{5} 1024$ The primary outcome of interest for many of these interventions is 30-day acute care readmission rates, since this metric is an indicator of quality of care and is tied to acute care financial incentives in some delivery systems. ${ }^{10}$ The two most widely disseminated models of care transition are Coleman $e t a l$ s and Naylor and colleagues' $(1993,2004)$ interventions. ${ }^{6} \quad{ }^{25-27}$ Coleman's care transition intervention uses a dedicated healthcare provider as a transition coach to help patients self-care in four areas posthospital discharge: medication management, understanding potential problems or 'red flags', scheduling follow-up care and engaging with providers by asking about their conditions. ${ }^{25}$ Naylor's model employs a dedicated nursing professional (eg, advanced practice nurse) to help patients self-care (similarly to Coleman's model), and also includes provision of a formalised follow-up care plan and regular home visits and telephone calls. ${ }^{2527}$ According to their landmark randomised controlled trials, both Coleman's and Naylor's models resulted in a $30-50 \%$ decrease in hospital readmissions. ${ }^{6} 2627$ Furthermore, Naylor's care transition intervention has been effective when applied specifically to elderly patients who are high-cost users and/or at high risk for hospital readmission. ${ }^{6} 2527$

Other care transition models, some of which were adapted from either Coleman's or Naylor's model, have been developed and implemented in a variety of settings with varying effectiveness. ${ }^{5} 102428$ A 2011 systematic review by Hansen and colleagues compared and summarised 43 studies that evaluated the effectiveness of interventions aimed at reducing 30-day hospital readmissions. ${ }^{24}$ Hansen $e t a l^{24}$ concluded that either was it not possible to determine effectiveness of specific components of multicomponent interventions, or was it possible to identify an intervention design that was consistently effective across studies due to the heterogeneity of both intervention components and the possible influence of context. Similar results were found by Naylor et a $a \tilde{t}$ in a systematic review of 21 randomised controlled trials for care transitions in older chronically ill adults, and Hesselink et al $\mathrm{s}^{12}$ systematic review of 36 studies for care transitions from acute care discharge to primary care. ${ }^{5} 29$ These findings suggest that some models or components of models are only effective within certain contexts, and that the current evaluations of these models have limited utility in deciding which intervention are best suited to particular patient groups or care environments. ${ }^{24} 30$

Ultimately, these mixed findings may result in stakeholders, such as hospital administrators and policymakers, implementing seemingly effective interventions into contexts where they may not actually be effective. Although Burke and Coleman ${ }^{31}$ recently outlined five best practice principles for implementing cost-effective interventions, there is very little evidence regarding why certain transition interventions work in some settings (ie, certain contexts) but not in others. In essence, there is a need to unpack how and within which contexts care transition interventions and their components are effective.

Realist reviews are a synthesis method popularised in the UK by Pawson and colleagues in 2004. ${ }^{32}$ Realist reviews differ from more traditional synthesis methods, such as scoping and systematic reviews, because they explain how complex programmes work or do not work within various contexts by exploring context, mechanisms and outcomes in relation to a programme's activities. ${ }^{33}$ They are grounded by the generative model of causality, meaning that to infer a causal relationship between an intervention and an outcome, the mechanism(s), the context, and the interaction between them must be understood.$^{33}$ Within the past decade, realist reviews have been conducted to explore a number of complex healthcare interventions, including patientreported outcome measures, self-management for chronically ill patients, and the relationship between poverty and health outcomes. ${ }^{34-36}$

The purpose of this study is to explain the effect of context on the activities and mechanisms of care transition interventions in medically complex older adults using a realist review approach. The overarching research question guiding this review is "What are the activities and underlying mechanisms in care transition interventions and how does context influence their role?”.

\section{METHODS AND ANALYSIS}

\section{Overall approach and theories of care transitions}

This synthesis will be guided by Pawson and colleagues' 2004 and 2005 protocols for conducting realist reviews, and reporting standards will follow Wong et $a l^{37}$ Realist and Meta-narrative Evidence Syntheses: Evolving Standards (RAMESES). ${ }^{33}$ Pawson's five steps for conducting realist review will be followed: (1) clarifying the scope of the review; (2) determining the search strategy; (3) study selection; (4) extracting data and (5) synthesising the evidence and drawing conclusions. ${ }^{32}$

The underlying theories of care transition interventions were determined based on an initial literature search using relevant databases (eg, MEDLINE (OVID 
interface), CINAHL [EBSCO interface)) conducted by two study team members (KBP and NEL) ${ }^{38}$ Underlying theories were defined as the programme theories (ie, 'small theories') that when combined, form the overarching programme theory of care transition interventions. ${ }^{32} 39$ This initial search found that the Coleman's ${ }^{40}$ and Naylor's (2004) model of care transition interventions (described above) are widely disseminated. The programme theory that emerges from these two interventions suggests that by providing support before, during and after the transition from one provider and/ or institution to another, patients will receive timely and appropriate care, resulting in decreased likelihood of adverse events. For the purpose of this study, a care transition intervention will be operationalised as any predischarge, post-discharge or bridging programme that is targeted at improving the transition from hospital discharge to home-based care management. ${ }^{24}$

We found that underlying theories in care transition interventions generally hypothesised a relationship between specific activities and outcomes, via a set of mechanisms that functioned differently across different contexts. ${ }^{6} 263340$

For the purpose of this study, context is defined as the organisational or environmental 'back-drop' of a care transition intervention that triggers or modifies actions of activities (eg, strength of networks between hospital staff and community-based providers). ${ }^{41}$ Activities are defined as the processes, tools, events, technology and actions that are an intentional part of the programme implementation. ${ }^{42}$ The definition of a mechanism is borrowed from Astbury and Leeuw, ${ }^{43}$ who define mechanisms as "underlying entities, processes, or structures which operate in particular contexts to generate outcomes of interest." ${ }^{43}$ Astbury and Leeuw identify three key characteristics of mechanisms: (1) mechanisms are usually hidden; (2) mechanisms are sensitive to variations in contexts and (3) mechanisms generate outcomes. $^{43}$ Outcomes are defined as changes in programme participants' healthcare utilisation, subjective or objective health status knowledge, skills, satisfaction or other outcomes as a result of intervention activities occurring in a given context. ${ }^{41}$ These include both intended and unintended outcomes of the intervention. $^{41}$

This review will use realist synthesis to unpack the Context-Activity-Mechanism-Outcome (CAMO) configurations underlying these theories of transitional support. ${ }^{32}$ Unpacking these CAMO configurations will provide insight into which theories of transitional care work, how they work and in which contexts. ${ }^{33}$

\section{Scope of the review}

This review is limited to care transition interventions targeted at older complex patients who have been discharged from acute care to home. A hard cut-off age criteria will not be used to exclude studies, however we will be targeting studies that describe their populations as 'older patients', and may therefore include populations as young as aged 50 years and older. Interventions comprised solely of disease-specific activities (eg, measurement of brain natriuretic peptide before heart failure discharge) will be excluded. Interventions comprising of only one activity (eg, medication reconciliation only) will also be excluded. These exclusion criteria were chosen for two reasons: first, these types of interventions are likely targeting a different population than our population of interest (ie, not necessarily complex older adults); second, one aspect of interest about the impact of context in care transitions is how context impacts the interaction or added impact of intervention activities. All study designs will be included (eg, observational studies, randomised controlled trials, qualitative studies). Only English language peer-reviewed studies published after 1993 will be included. Despite the potential differences from the current healthcare contexts, studies published from 1993 onward were included to ensure studies published in the era of Naylor $e t a l^{27}$ randomised controlled trial were captured. ${ }^{27}$

\section{Search strategy and study selection}

The search strategy was developed and will be implemented in collaboration with an experienced information scientist. MEDLINE (OVID interface), EMBASE (OVID interface), CINAHL (EBSCO interface), AgeLine (EBSCO interface) and Cochrane Central Register Controlled Trials (Cochrane Library) databases will be searched using medical subject headings (MeSH) and text terms related to care transitions (including synonyms such as intermediate care) for older adults. ${ }^{23}$ Initial development of the search strategy yielded the following MeSH terms: 'Patient Discharge' OR 'post discharge' OR 'postdischarge' OR 'after care' AND 'continuity of patient care' OR 'care transition' OR 'discharge planning' OR 'discharge plan'. The search strategy will be reviewed iteratively by the study team to ensure the scope is adequate to answer the overarching research question.

Not surprisingly, Hansen et al $\mathrm{s}^{24}$ systematic review of care transition interventions that reduce 30-day re-hospitalisation concluded that the studies reviewed did not adequately describe interventional components or context sufficiently for cross-study comparisons to be made. ${ }^{24}$ It is therefore acknowledged that detailed information on certain aspects of interventions that may be pertinent to answering our overarching research question, particularly information on contextual factors and mechanisms, is likely not reported. To extract this relevant but unpublished information, a search for associated and relevant publications will be conducted, if required, using the following steps: (1) a Scopus search of the initial publication followed by examination of all citing manuscripts; (2) PubMed (OVID interface) search using the corresponding author's name; (3) the first two pages of a Google search for the name of the study (for possible grey literature) and (4) a review of 
the Corresponding Author's ResearchGate publications (to capture unpublished information such as abstracts). If pertinent information is still unavailable, corresponding authors will be contacted via email or telephone.

\section{Data collection}

A study inclusion screening form will be developed and piloted by two reviewers (KBP and NEL) on approximately $1 \%$ of articles. Titles and abstracts will then be screened using the form for potentially relevant articles (level 1 screening). Another screening form for full-text review will then be piloted on approximately $1 \%$ of articles by two reviewers (KBP and NEL). Full text of potentially relevant articles will then be retrieved and screened to determine final inclusion criteria (level 2 screening). The reasons for exclusion will be recorded. All studies at both levels 1 and 2 will be screened in duplicate by two reviewers (TV and AIK) to increase reliability of the application of the study inclusion criteria. ${ }^{33} 3844 \quad 45$ Any discrepancies will be resolved by a third reviewer (JI) who is knowledgeable in the field of care transition interventions.

Abstracted data will include study characteristics (eg, publication year, study design); intervention type (eg, bridging, pre-discharge); contextual factors (eg, study setting, designation of intervention staff (eg, nurse), dedicated programme staff (yes/no)); intervention activities (eg, medication reconciliation); and underlying mechanisms (eg, relationship development). Study thickness will be determined to help inform the study's quality and therefore the relative weight its' results should be given during data synthesis. ${ }^{23}{ }^{46}$ Information on study rigour (eg, trustworthiness of methods) will also be noted. ${ }^{32-34}$

\section{Synthesising the evidence}

Study characteristics, target populations and a high-level description of each care transition intervention will be summarised by intervention type (ie, pre-discharge, bridging or post-discharge intervention). CAMO configurations will then be identified for each study with the ultimate goal of determining how each care transition activity works or does not work and within which contexts. ${ }^{34}$ These configurations will be iteratively created by two study team members and refined by the entire study team throughout the synthesis process. ${ }^{34}$ First, CAMO chains will be examined to see which patterns are recurrent. ${ }^{34}$ These recurrent patterns and mechanisms will then be inspected to identify potential CAMO configurations. ${ }^{34}$ If information is conflicting between studies, priority will be given to studies that have described putative mechanisms in-depth. ${ }^{34}$ Finalised recurrent CAMO configurations will then be reported with the intended purpose of highlighting the complexity (ie, breadth and variety) of existing programme theories of care transition, or perhaps discovering new programme theories (ie, 'small theories'), thereby providing stakeholders with guidance as to what to consider when implementing these complex interventions. ${ }^{33}$ The delivery of healthcare has changed over the study time period, and the impact of this change in relation to study findings will be discussed.

\section{ETHICS AND DISSEMINATION}

Ethics is not required for this realist review. Internal knowledge translation activities (eg, within the Health System Performance Research Network) will occur throughout the review to gather experts' opinions on data analyses and to create general awareness of the project. Existing partnerships with acute care institutions (eg, University Health Network) and the Ontario Ministry of Health and Long-Term Care will be leveraged to disseminate findings to frontline staff, hospital administrators, and policymakers. Finalised results will be presented at local (eg, Health Quality Ontario), national (eg, Canadian Association of Health Service and Policy Research) and international (eg, AcademyHealth Annual Research Meeting) conferences, and disseminated via peer-reviewed publications in relevant journals.

This realist review will address an existing knowledge gap by summarising evidence on how context impacts activities, mechanisms and effectiveness of care transition interventions. By understanding how these interventions work and how context impacts their effectiveness, stakeholders can make evidence-based decisions on which interventions to implement within their local context.

Twitter Follow Kristen Pitzul at @kpitzul, Natasha Lane at @NatashaErinLane, Jennifer Innis at @JenniferInnisNP, Walter Wodchis at @wwodchis, and Ross Baker at @baker_ross

Contributors KBP was responsible for project conception, protocol development, writing and submission of the manuscript. NEL and GRB were responsible for project conception, protocol development and editing of the manuscript. TV, AIK, JI and WPW were responsible for protocol development and editing of the manuscript.

Funding This work was supported by the Health Systems Performance Research Network (HSPRN), which is funded by a network grant from the Ontario Ministry of Health and Long-Term Care (MOHLTC grant \#06034).

Competing interests None declared.

Provenance and peer review Not commissioned; externally peer reviewed.

Open Access This is an Open Access article distributed in accordance with the Creative Commons Attribution Non Commercial (CC BY-NC 4.0) license, which permits others to distribute, remix, adapt, build upon this work noncommercially, and license their derivative works on different terms, provided the original work is properly cited and the use is non-commercial. See: http:// creativecommons.org/licenses/by-nc/4.0/

\section{REFERENCES}

1. Kinsella K, Velkoff VA. International population reports. Washington DC: U.S. Census Bureau, 2001.

2. Lochner KA, Cox CS. Prevalence of multiple chronic conditions among medicare beneficiaries, United States, 2010. Prevalence Chron Dis 2013;10:120-37.

3. Turcotte M, Schellenberg G. A portrait of seniors in Canada. Statistics Canada. Ottawa, Ontario, 2007.

4. Coleman EA, Boult C. Improving the quality of transitional care for persons with complex care needs. J Am Geriatr Soc 2003;51:556-7. 
5. Naylor MD, Aiken LH, Kurtzman ET, et al. The care span: the importance of transitional care in achieving health reform. Health Aff (Millwood) 2011;30:746-54.

6. Naylor MD, Brooten DA, Campbell RL, et al. Transitional care of older adults hospitalized with heart failure: a randomized, controlled trial. J Am Geriatr Soc 2004;52:675-84.

7. IOM. Crossing the quality chasm: a new health system for the 21st century. Washington DC: National Academy Press, 2001.

8. CIHI. Seniors and the health care system: what is the impact of multiple chronic conditions? Ottawa, ON: Canadian Institute of Health Information, 2011

9. Schuster MA, McGlynn EA, Brook RH. How good is the quality of health care in the United States? Milbank Q 2005;83:843-95.

10. Kim CS, Flanders SA. Transitions of care. Ann Intern Med 2013; 158 (5 Part 1):ITC3-1.

11. Kripalani S, LeFevre F, Phillips CO, et al. Deficits in communication and information transfer between hospital-based and primary care physicians: implications for patient safety and continuity of care. JAMA 2007;297:831-41.

12. Hesselink G, Vernooij-Dassen M, Pijnenborg L, et al. Organizationa culture: an important context for addressing and improving hospital to community patient discharge. Med Care 2013;51:90-8.

13. Jencks SF, Williams MV, Coleman EA. Re-hospitalizations among patients in the Medicare fee-for-service program. $N$ Engl J Med 2009;360:1418-28.

14. LaMantia MA, Scheunemann LP, Viera AJ, et al. Interventions to improve transitional care between nursing homes and hospitals: a systematic review. J Am Geriatr Soc 2010;58:777-82.

15. Gandara E, Moniz T, Ungar J, et al. Communication and information deficits in patients discharged to rehabilitation facilities: an evaluation of five acute care hospitals. J Hosp Med 2009;4:E28-33.

16. Weaver FM, Perloff L, Waters T. Patients' and caregivers' transition from hospital to home. Needs and recommendations. Home Health Care Serv Q 1998;17:27-48.

17. Hendriks AA, Vrielink MR, Smets EM, et al. Improving the assessment of (in)patients' satisfaction with hospital care. Med Care 2001;39:270-83.

18. Forster AJ, Murff HJ, Peterson JF, et al. The incidence and severity of adverse events affecting patients after discharge from the hospital. Ann Intern Med 2003;138:161-7.

19. Forster AJ, Clark HD, Menard A, et al. Adverse events among medical patients after discharge from hospital. CMAJ 2004;170:345-9.

20. Corbett CF, Setter SM, Daratha KB, et al. Nurse identified hospital to home medication discrepancies: implications for improving transitional care. Geriatr Nurs 2010;31:188-96.

21. Tsilimingras $D$, Bates DW. Addressing postdischarge adverse events: a neglected area. Jt Comm J Qual Patient Saf 2008;34:85-97.

22. Schoen C, Osborn R, How SK, et al. In chronic condition: experiences of patients with complex health care needs, in eight countries, 2008. Health Aff (Millwood) 2009;1:w1-w16.

23. Pearson $M$, Hunt $\mathrm{H}$, Cooper $\mathrm{C}$, et al. Providing effective and preferred care closer to home: a realist review of intermediate care. Health Soc Care Community 2015;3:577-93.

24. Hansen LO, Young RS, Hinami K, et al. . Interventions to reduce 30-day rehospitalization: a systematic review. Ann Inter Med 2011;155:520-8.

25. Burton R. Health policy brief: improving care transitions. Health Affairs September 13th 2012.
26. Coleman EA, Parry C, Chalmers S, et al. The care transitions intervention: results of a randomized controlled trial. Arch Intern Med 2006;166:1822-8.

27. Naylor M, Brooten DA, Jones $\mathrm{R}$, et al. Comprehensive discharge planning for the hospitalized elderly: a randomized clinical trial. Ann Intern Med 1994;120:999-1006.

28. Jack BW, Chetty VK, Anthony D, et al. A reengineered hospital discharge program to decrease rehospitalization: a randomized trial. Ann Intern Med 2009;150:178-87.

29. Hesselink G, Schoonhoven L, Barach $P$, et al. Improving patient handovers from hospital to primary care. Ann Intern Med 2012;157:417-28.

30. Gray LC, Peel NM, Crotty M, et al. How effective are programs a managing transition from hospital to home? A case study of the Australian transition care program. BMC Geriatrics 2012;12:1-5.

31. Burke RE, Coleman EA. Interventions to decrease hospital readmissions. J Am Med Assoc Intern Med 2013;173:695-8.

32. Pawson R, Greenhalgh T, Harvey G, et al. Realist synthesis: an introduction ESRC research methods programme. Manchester, UK: University of Manchester, 2004.

33. Pawson R, Greenhalgh T, Harvey G, et al. Realist review-a new method of systematic review designed for complex policy interventinos. J Health Serv Res Policy 2005;10(Suppl):21-34.

34. Molnar A, O'Campo P, Ng E, et al. Protocol: realist synthesis of the impact of unemployment insurance policies on poverty and health. Eval Program Plan 2015;48:1-9.

35. Mills SL, Pumarino J, Clark N, et al. Understanding how self-management interventions work for disadvantaged populations living with chronic conditions: protocol for a realist synthesis. BMJ Open 2014;4:e005822.

36. Greenhalgh J, Pawson R, Wright J, et al. Functionality and feedback: a protocol for a realist synthesis of the collation, interpretation and utilisation of PROMS data to improve patient care. BMJ Open 2014;4:e005601.

37. Wong G, Greenhalgh T, Westhorp G, et al. RAMESES publication standards: realist syntheses. BMC Med 2013;11:21.

38. Arksey H, O'Malley L. Scoping studies: towards a methodologica framework. Int J Soc Res Methodol 2005;8:19-32.

39. Davidoff F, Dixon-Woods M, Leviton L, et al. Demystifying theory and its use in improvement. BMJ Qual Saf 2015;24:228-38.

40. Coleman EA. Falling through the cracks: challenges and opportunities for improving transitional care for persons with continuous complex care needs. Am Geriatr Soc 2003;51:549-55.

41. Jagosh J, Macaulay AC, Pluye P, et al. Uncovering the benefits of participatory research: implications of a realist review for health research and practice. Milbank Q 2012;90:311-46.

42. Foundation WKK. W.K. Kellogg Foundation Logic Model Development Guide. Chapters 2 and 3. 2004. http://www.epa.gov/ evaluate/pdf/eval-guides/logic-model-development-guide.pdf

43. Astbury B, Leeuw FL. Unpacking black boxes: mechanisms and theory building in evaluation. Am J Eval 2010;31:363-81.

44. Cochrane. Cochrane Reviewers' Handbook. Cochrane; March 2005 2004.

45. Levac DC, Colquhoun H, O'Brien KK. Scoping studies: advancing the methodology. Implement Sci 2010;5:69.

46. Roen K, Arai L, Roberts $\mathrm{H}$, et al. Extending systematic reviews to include evidence on implementation: methodological work on a review of community-based initiatives to prevent injuries. Soc $\mathrm{Sci}$ Med 2006;63:1060-71. 